\title{
Classification of posterior vitreous detachment
}

This article was published in the following Dove Press journal:

Clinical Ophthalmology

3 December 2013

Number of times this article has been viewed

\author{
Akihiro Kakehashi' \\ Mikiko Takezawa' \\ Jun Akiba ${ }^{2}$ \\ 'Department of Ophthalmology, \\ Jichi Medical University, Saitama \\ Medical Center, Saitama, ${ }^{2}$ Kanjodori \\ Eye Clinic, Asahikawa, Japan
}

Correspondence: Akihiro Kakehashi Department of Ophthalmology, Jichi Medical University, Saitama Medical Center, I-847 Amanuma-cho, Omiya-ku, Saitama-shi, 330-8503, Japan

Tel +8 I 486472 III

$\mathrm{Fax}+8 \mid 6485188$

Email kakeaki@omiya.jichi.ac.jp
Abstract: Diagnosing a posterior vitreous detachment (PVD) is important for predicting the prognosis and determining the indication for vitreoretinal surgery in many vitreoretinal diseases. This article presents both classifications of a PVD by slit-lamp biomicroscopy and of a shallow PVD by optical coherence tomography (OCT). By biomicroscopy, the vitreous condition is determined based on the presence or absence of a PVD. The PVD then is classified as either a complete posterior vitreous detachment (C-PVD) or a partial posterior vitreous detachment (P-PVD). A C-PVD is further divided into a C-PVD with collapse and a C-PVD without collapse, while a P-PVD is divided into a P-PVD with shrinkage of the posterior hyaloid membrane (P-PVD with shrinkage) and a P-PVD without shrinkage of the posterior hyaloid membrane (P-PVD without shrinkage). A P-PVD without shrinkage has a subtype characterized by vitreous gel attachment through the premacular hole in a posterior hyaloid membrane to the macula (P-PVD without shrinkage [M]). By OCT, a shallow PVD is classified as the absence of a shallow PVD or as a shallow PVD. A shallow PVD is then subclassified as a shallow PVD without shrinkage of the posterior vitreous cortex, a shallow PVD with shrinkage of the posterior vitreous cortex, and a peripheral shallow PVD. A shallow PVD without shrinkage of the posterior vitreous cortex has two subtypes: an age-related shallow PVD and a perifoveal PVD associated with a macular hole.

Keywords: classification, optical coherence tomography, PVD, slit-lamp biomicroscopy

\section{Introduction}

Diagnosing vitreous changes, especially a posterior vitreous detachment (PVD), is important for predicting the prognosis and determining the indication for vitreoretinal surgery in many vitreoretinal diseases. In diabetic retinopathy, a partial posterior vitreous detachment (P-PVD) or the absence of a PVD is a risk factor for retinal neovascularization, but a complete posterior vitreous detachment (C-PVD) is a strong negative risk factor for retinal neovascularization. ${ }^{1,2}$ Vitreous attachment to the retina is the scaffold for neovascularization arising from the retina. Retinal neovascularization never arises from the retina in eyes with a C-PVD.

In certain cases with diabetic macular edema (DME), vitreous traction or attachment to the macula worsens the macular edema. ${ }^{3}$ In these cases, vitreous surgery is indicated in addition to laser treatment or intravitreous injection of an anti-vascular endothelial growth factor drug or triamcinolone.

Rhegmatogenous retinal detachments due to retinal holes in the lattice degeneration in eyes without a PVD, usually found in young patients, progress slowly. However, because rhegmatogenous retinal detachments due to retinal tears associated with a PVD progress rapidly, emergency surgery is needed. 
Optical coherence tomography (OCT) can visualize perifoveal PVDs with pinpoint vitreofoveal traction in cases with an idiopathic macular hole, but biomicroscopy cannot. ${ }^{4} \mathrm{~A}$ macular hole does not develop in the fellow eye of one with a macular hole when there is a shallow PVD over the fovea, even if there is no evidence of a PVD by biomicroscopy.

There are three primary methods for detecting a PVD: biomicroscopy, ultrasonography, and OCT. Ultrasonography is the first choice to detect a PVD in cases with media opaque. This article presents classifications of PVDs by slit-lamp biomicroscopy and classifications of shallow PVDs by OCT.

\section{Examination technique to detect PVDs Preparation for vitreoretinal examination}

Before slit-lamp biomicroscopy, the pupil should be dilated fully to obtain a wide illumination-observation angle for optical sections of the vitreous. To obtain a sharp optical section of the vitreous, slit illumination should be set narrow and be the brightest possible. We usually use the SL 130 Slit Lamp (Carl Zeiss Meditec AG, Jena, Germany) or Slit Lamp BM $900^{\circledR}$ with a stereo validator (Haag-Streit, Bern, Switzerland) slit-lamp biomicroscope for biomicroscopic vitreous examinations. Three types of precorneal lens - the Goldmann three-mirror contact lens, the non-contact positive preset lens, and the wide-angle funduscopic contact lens - should be available for a comprehensive biomicroscopic vitreous examination. In most cases, the posterior vitreous can be examined using a non-contact positive preset lens such as the SuperField NC Lens (Volk, Mentor, OH, USA). The technique of biomicroscopic vitreous examination has been previously published in detail. ${ }^{5}$

For OCT vitreoretinal examinations, pupil dilation is preferred but not needed in all cases. Time-domain (TD) OCT does not have sufficient resolution to evaluate the minute changes in the vitreoretinal interface. Spectral-domain OCT (SD-OCT) is needed to evaluate the morphologic changes in the vitreoretinal interface. We usually use a Cirrus HD OCT (Carl Zeiss Meditec) and select the 9-mm five-line raster mode including the optic disc and the macula.

\section{Classification of PVDs Classification of a PVD by slit-lamp biomicroscopy}

The classification of PVDs by slit-lamp biomicroscopy is shown in Figure 1 and Table 1. First, the vitreous condition is classified according to the presence or absence of a PVD. The PVD then is subclassified as a C-PVD or a P-PVD. The C-PVD is further divided into a C-PVD with and without collapse, while a P-PVD is divided into a P-PVD with and without shrinkage of the posterior hyaloid membrane (P-PVD with shrinkage and P-PVD without shrinkage, respectively). A P-PVD without shrinkage has a subtype characterized by vitreous gel attachment through the premacular hole in a posterior hyaloid membrane to the macula (P-PVD without shrinkage[M]).

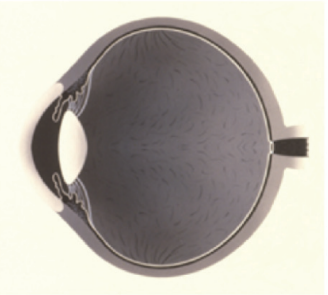

No PVD

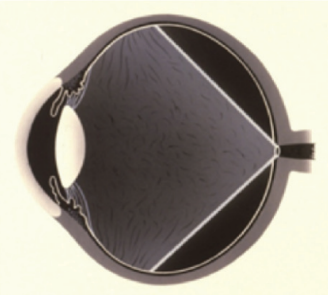

P-PVD with shrinkage

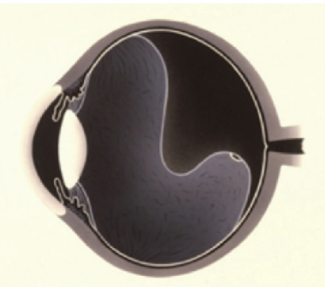

C-PVD with collapse

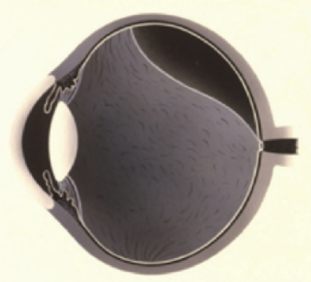

P-PVD without shrinkage

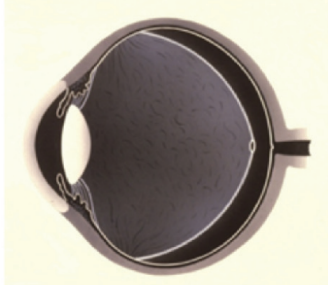

C-PVD without collapse

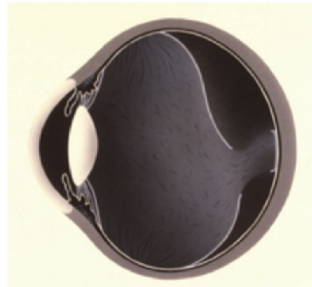

P-PVD without shrinkage (M)

Figure I Diagram of the posterior vitreous detachment classification by slit-lamp biomicroscopy.

Abbreviations: C-PVD, complete posterior vitreous detachment; M, vitreous attachment to the macula; P-PVD, partial posterior vitreous detachment. 
Table I Classification of a posterior vitreous detachment by slitlamp biomicroscopy

\begin{tabular}{llll}
\hline PVD & & \\
\hline C-PVD & & P-PVD & \\
\hline C-PVD with & C-PVD without & P-PVD with & P-PVD without \\
collapse & collapse & shrinkage & $\begin{array}{l}\text { shrinkage } \\
\text { P-PVD without } \\
\end{array}$ \\
& & & shrinkage (M) \\
\hline
\end{tabular}

Abbreviations: C-PVD, complete posterior vitreous detachment; M, vitreous attachment to the macula; P-PVD, partial posterior vitreous detachment.

The biomicroscopic findings for each type of PVD are presented as follows. Case 1 (Figure 2, Video 1 http://www. youtube.com/watch? $\mathrm{v}=\mathrm{GPRCsXarBMY}$ ) shows asteroid hyalosis, which facilitates diagnosing the absence of a PVD. In this case, the posterior vitreous did not separate from the retina even after ocular movement.

Case 2 (Figure 3, Video 2 http://www.youtube.com/ watch? $\mathrm{v}=\mathrm{H} 2 \mathrm{hWjOIXOJA}$ ) shows a normal eye without a PVD. There was no evidence of vitreous opacity by indirect ophthalmoscopy. Although it was difficult to determine the absence of a PVD when compared with Case 1, the Tyndall phenomenon in the slit section of the vitreous and no evidence of a posterior hyaloid membrane with a prepapillary glial ring even after ocular movement established the absence of a PVD.

Case 3 (Figure 4, Video 3 http://www.youtube.com/ watch? $\mathrm{v}=\mathrm{M} 0 \mathrm{hl}-\mathrm{SY} \quad \mathrm{OSQ}$ ) is a normal eye with a C-PVD with collapse. A mobile, highly detached posterior hyaloid membrane was traced easily and a prepapillary glial ring was also observed on the posterior hyaloid membrane, making it easy to diagnose a C-PVD with collapse.

Case 4 (Figure 5, Video 4 http://www.youtube.com/ watch? $v=$ AaaMAkrD57M) had diabetic retinopathy after panretinal photocoagulation with a C-PVD without collapse. A shallowly detached posterior hyaloid membrane was traced just

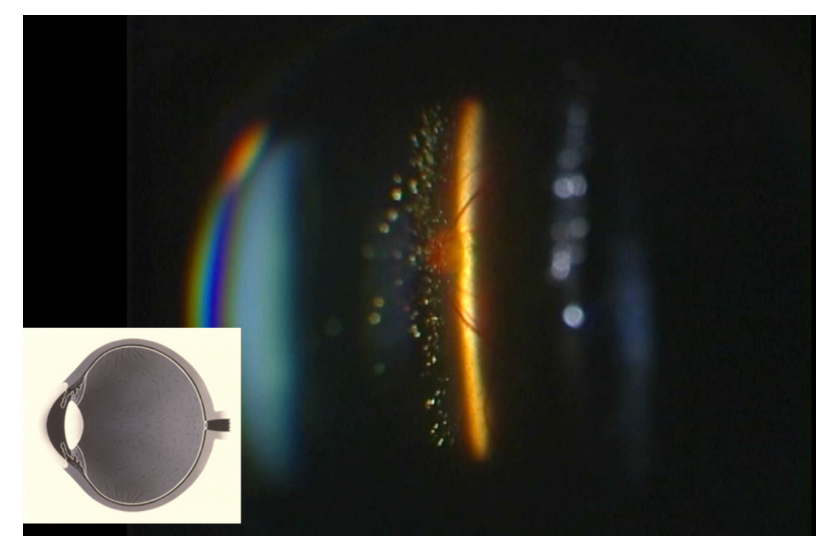

Figure 2 The absence of a posterior vitreous detachment (PVD). Case I shows asteroid hyalosis with no PVD. The posterior vitreous is not separated from the retina even after ocular movement. Many asteroid bodies can be observed in the vitreous gel.

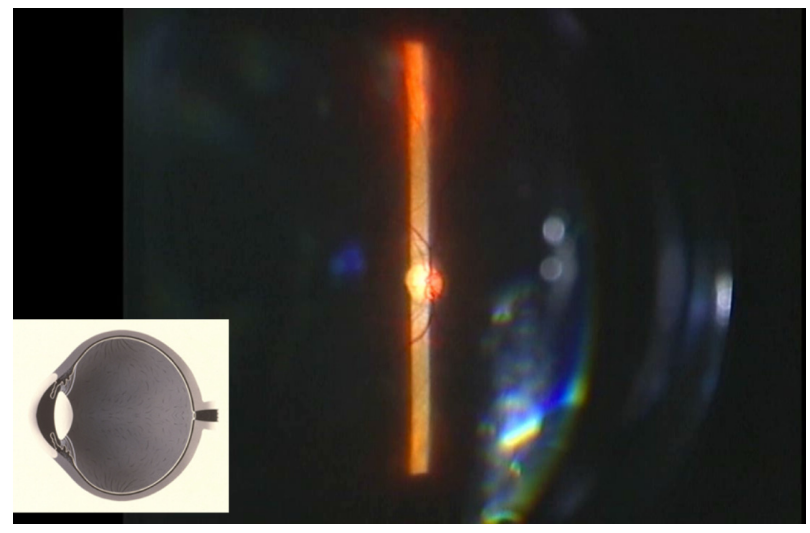

Figure 3 The absence of a posterior vitreous detachment (PVD). A normal eye with no PVD. The Tyndall phenomenon in the slit section of the vitreous and no evidence of a posterior hyaloid membrane with a prepapillary glial ring even after ocular movement results in a diagnosis of no PVD in this case.

in front of the retina and then diagnosed as a C-PVD without collapse. The eyes of young patients with central retinal vein occlusion and uveitis also frequently have this type of PVD.

Case 5 (Figure 6, Video 5 http://www.youtube.com/ watch? $v=-$ RsCnMDB7sc) had proliferative diabetic retinopathy with a P-PVD with shrinkage. Neovascular proliferative tissue was observed along the retinal vascular arcade. A posterior hyaloid membrane, including the neovascular proliferative tissue, was not mobile even after ocular movement. This vitreous change caused strong vitreous traction to the retina along the retinal vascular arcade.

Case 6 (Figure 7, Video 6 http://www.youtube.com/ watch? $=$ =ftMPOS5q2Co) shows a normal eye with a P-PVD without shrinkage. A mobile detached posterior hyaloid membrane was traced superiorly (inverted image). However, the inferior vitreous did not detach from the inferior retina even after ocular movement. Most cases with this type of a PVD are thought to be in a transient phase that will evolve into a C-PVD with collapse.

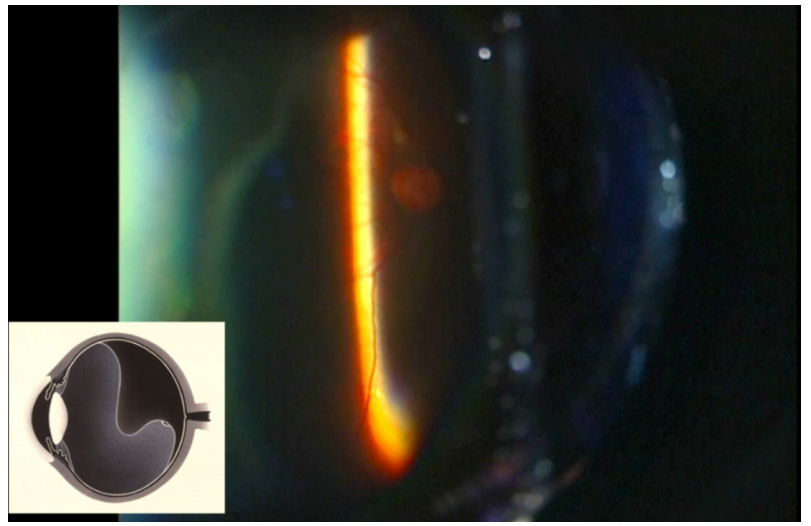

Figure $4 \mathrm{~A}$ complete posterior vitreous detachment with collapse in a normal eye. A mobile highly detached posterior hyaloid membrane can be traced easily and a prepapillary glial ring is observed on the posterior hyaloid membrane. 


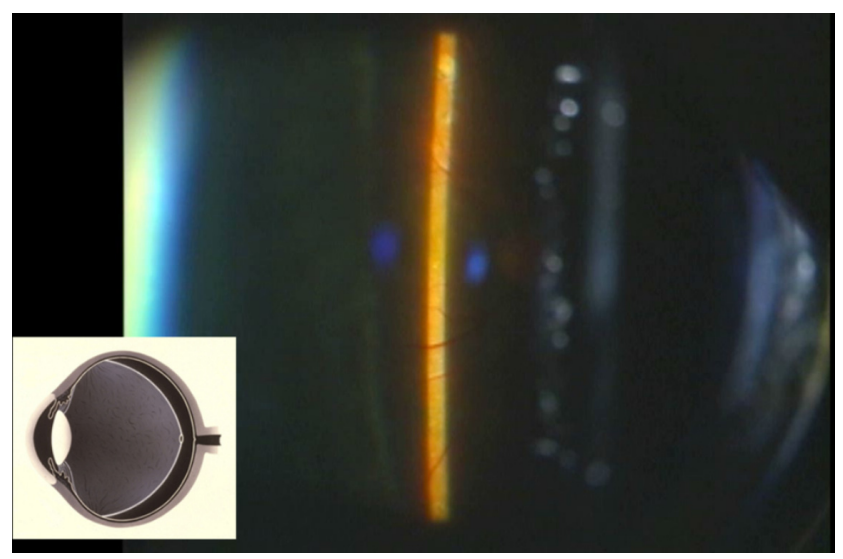

Figure 5 A complete posterior vitreous detachment (C-PVD) without collapse. Diabetic retinopathy after panretinal photocoagulation with a C-PVD without collapse. A shallowly detached posterior hyaloid membrane can be traced just in front of the retina and diagnosed as a C-PVD without collapse.

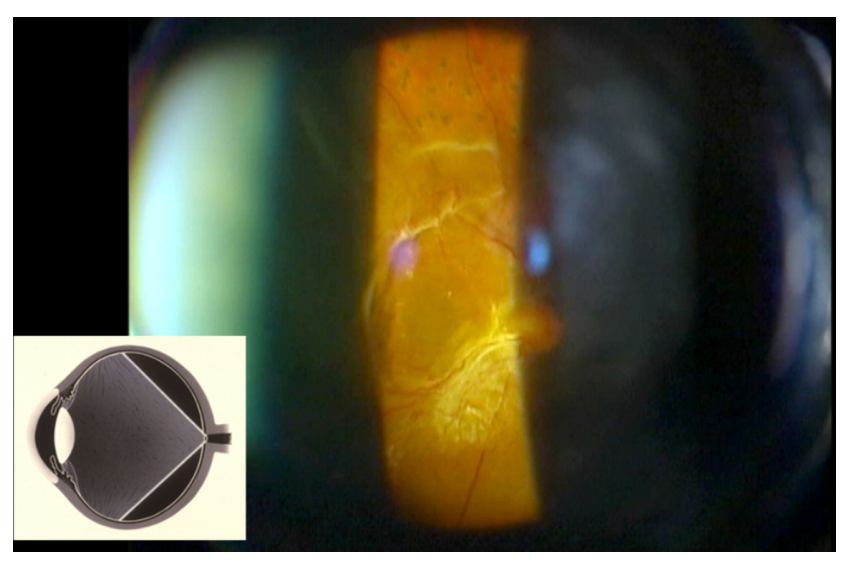

Figure 6 A partial posterior vitreous detachment (P-PVD) with shrinkage. Proliferative diabetic retinopathy with a P-PVD with shrinkage. Neovascular proliferative tissue can be observed along the retinal vascular arcade. A posterior hyaloid membrane including the neovascular proliferative tissue is not mobile even after ocular movement.

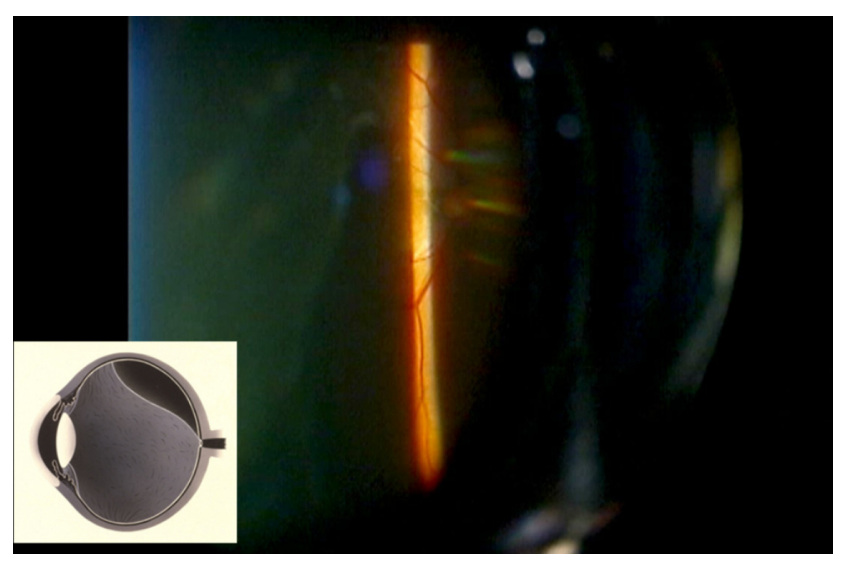

Figure 7 A partial posterior vitreous detachment without shrinkage in a normal eye. A mobile detached posterior hyaloid membrane can be traced superiorly in the inverted video image.

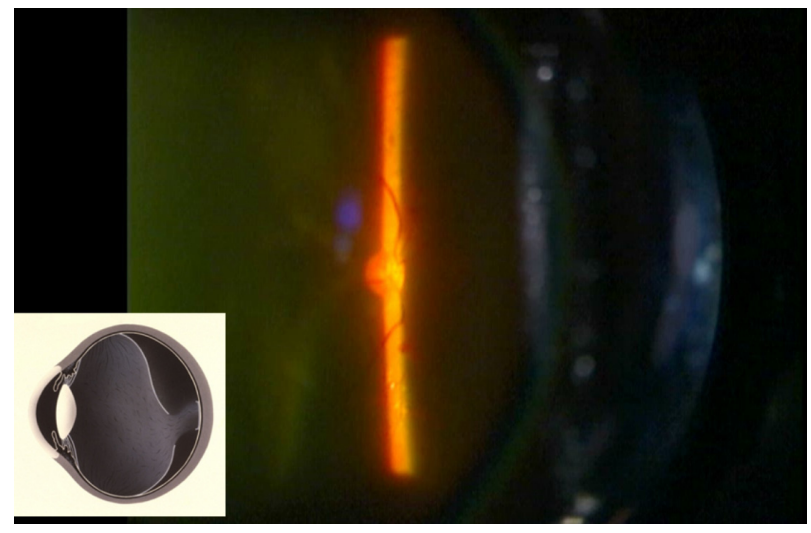

Figure 8 A partial posterior vitreous detachment (P-PVD) without shrinkage (M). An eye with diabetic macular edema with a P-PVD without shrinkage (M). Although most of the vitreous is detached from the retina, a detached posterior hyaloid membrane can be traced except for over the macula. The vitreous gel is attached through the premacular hole in the posterior hyaloid membrane.

Abbreviation: $M$, vitreous attachment to the macula.

Case 7 (Figure 8, Video 7 http://www.youtube.com/ watch? $v=n R K U P V F R w O Y$ ) had DME with a P-PVD without shrinkage. Although most of the vitreous was detached from the retina, a detached posterior hyaloid membrane was traced except for over the macula. The vitreous gel was attached through a premacular hole in the posterior hyaloid membrane, and this vitreous attachment caused weak but chronic vitreous traction on the macula. This vitreous change worsened the DME in this case. Although this vitreous pathology is somewhat difficult to detect by biomicroscopy, Sebag and Balazs clearly showed it using in vitro slit-lamp microscopy. ${ }^{6}$

\section{Classification of a shallow PVD by OCT}

TD-OCT does not have sufficient resolution to detect a shallow PVD and cannot detect a highly detached PVD. A previous study showed the inferiority of TD-OCT for detecting a PVD compared with ultrasonography and slitlamp-biomicroscopy. ${ }^{7}$ However, SD-OCT can detect a shallow PVD that is undetectable by slit-lamp biomicroscopy. Although some types of shallow PVDs can be detected by TD-OCT, ${ }^{8}$ various types of shallow PVDs and their associa-

Table 2 Classification of a shallow posterior vitreous detachment (PVD) by optical coherence tomography

\begin{tabular}{lcl}
\hline & Shallow PVD & \\
\hline $\begin{array}{l}\text { Shallow PVD without } \\
\text { shrinkage of posterior }\end{array}$ & $\begin{array}{l}\text { Shallow PVD with } \\
\text { shrinkage of posterior } \\
\text { vitreous cortex }\end{array}$ & $\begin{array}{l}\text { Peripheral } \\
\text { shallow }\end{array}$ \\
Age-related shallow PVD & & PVD \\
Shallow PVD without & & \\
shrinkage of posterior & & \\
vitreous cortex & & \\
Perifoveal PVD associated & & \\
with macular disease & & \\
\hline
\end{tabular}


A

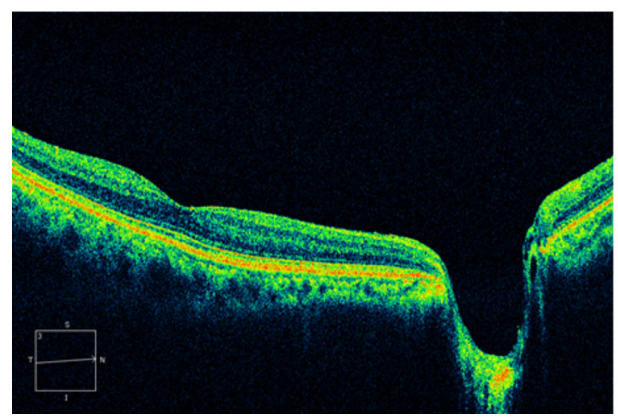

B

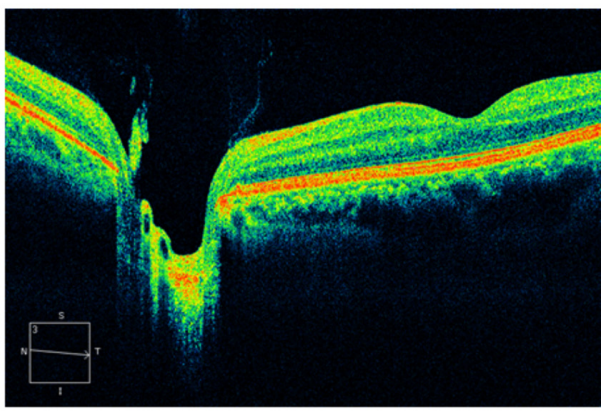

Figure 9 The absence of a shallow posterior vitreous detachment (PVD). (A) The case is diagnosed as absence of a PVD without evidence of Cloquet's canal by slit-lamp biomicroscopy. (B) Optical coherence tomography does not show a PVD because Cloquet's canal can be observed.

Note: Copyright @ 201 I, Dove Medical Press. Reproduced with permission from Takezawa M et al. Clarifying the mechanism of idiopathic macular hole development in fellow eyes using spectral-domain optical coherence tomography. Clin Ophthalmol. 20I I;5:10I-108. ${ }^{4}$

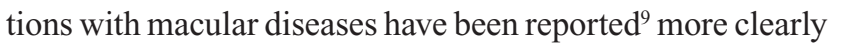
since the development of SD-OCT.

The classification of a shallow PVD seen using OCT is presented in Table 2. Slit-lamp biomicroscopy did not detect a shallow PVD that was observed by OCT. A shallow PVD is initially classified as the absence or presence of a shallow PVD.
The shallow PVD is then classified as a shallow PVD without shrinkage of the posterior vitreous cortex, a shallow PVD with shrinkage of the posterior vitreous cortex, or a peripheral shallow PVD. A shallow PVD without shrinkage of the posterior vitreous cortex has two subtypes: an age-related shallow PVD and a perifoveal PVD associated with a macular hole.
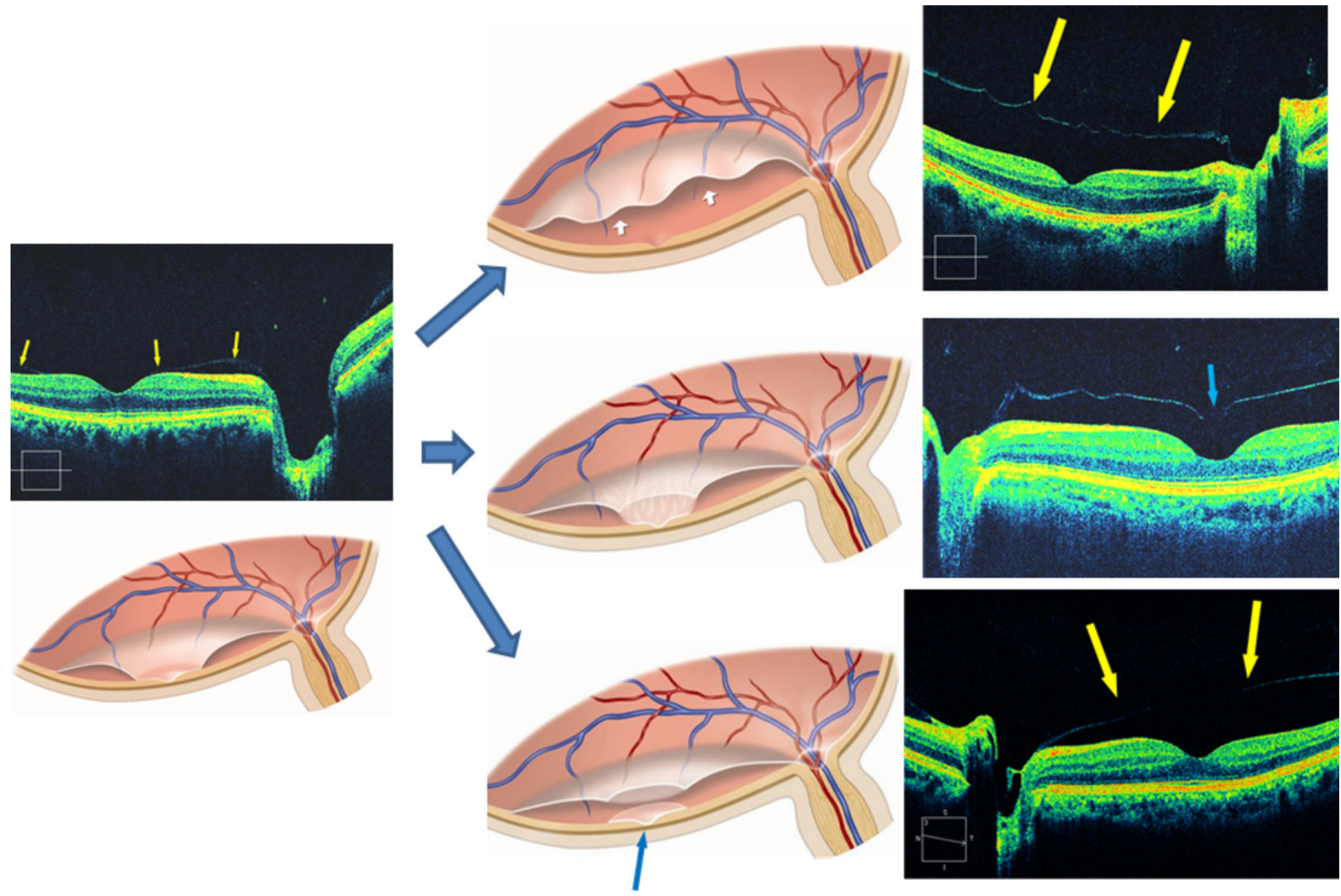

Figure 10 A shallow posterior vitreous detachment (PVD) without shrinkage of the posterior vitreous cortex. There are four variations of an age-related shallow PVD. In the left optical coherence tomography (OCT) image and diagram, a primarily shallow detached posterior vitreous cortex (arrows) is observed around the macula. This shallow PVD without shrinkage of the posterior vitreous cortex can progress to one of the other three types: (I) a shallow PVD without formation of a premacular hole in the posterior vitreous cortex (top right OCT image and diagram; the arrows in the diagram and in the OCT image indicate the detached posterior vitreous cortex); (2) a shallow PVD with formation of a premacular hole in the posterior vitreous cortex with vitreous gel attachment to the macula (middle right OCT image and diagram; the arrow in the OCT image indicates a premacular hole in the posterior vitreous cortex); or (3) a shallow PVD with formation of a premacular hole in the posterior vitreous cortex without vitreous gel attachment to the macula (bottom right OCT image and diagram; the arrow indicates a remnant of the posterior vitreous cortex over the macula and the arrows in the OCT image indicate the detached posterior vitreous cortex).

Note: Copyright (C) 20II, Dove Medical Press. Reproduced with permission from Takezawa M et al. Clarifying the mechanism of idiopathic macular hole development in fellow eyes using spectral-domain optical coherence tomography. Clin Ophthalmol. 20I I;5:10I-108. ${ }^{4}$ 


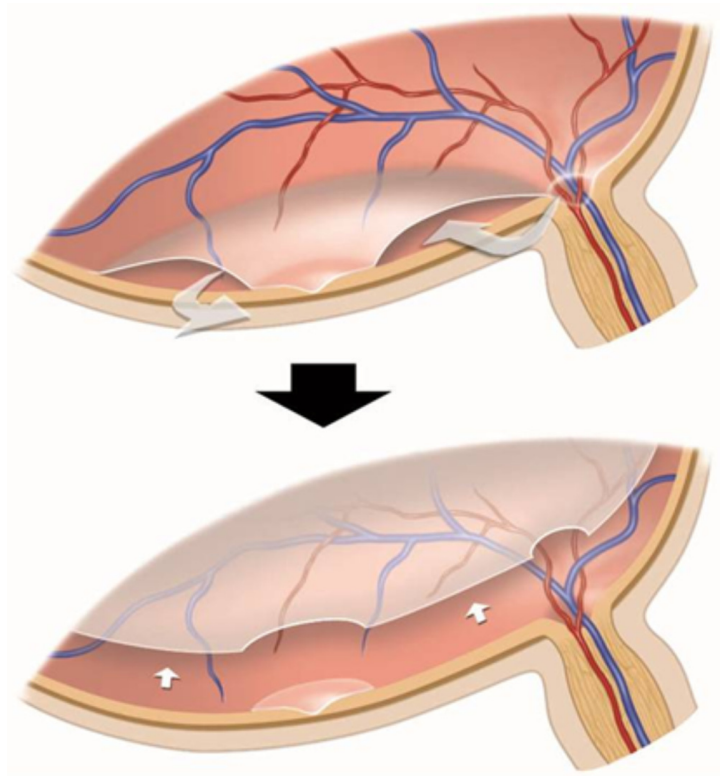

Figure I I Progression of a posterior vitreous detachment (PVD). In the primary stage of an age-related shallow PVD (top), liquefied vitreous (arrows) possibly enters the subhyaloid space via a crevice in the glial ring on the optic disc. When a premacular hole begins to form in the posterior vitreous cortex, a large amount of liquefied vitreous enters the subhyaloid space through the premacular hole in the posterior vitreous cortex, which induces the rapid formation of a high PVD (bottom, arrows) that can be detected by slit-lamp biomicroscopy but not optical coherence tomography.

Note: Copyright (C) 20II, Dove Medical Press. Reproduced with permission from Takezawa M et al. Clarifying the mechanism of idiopathic macular hole development in fellow eyes using spectral-domain optical coherence tomography. Clin Ophthalmol. 201।;5:101-108. ${ }^{4}$

The natural course of an age-related PVD is as follows. The absence of a PVD can be diagnosed by OCT when Cloquet's canal or vitreous cortex and gel over the posterior retina are observed. However, there are many cases of the absence of a PVD without evidence of Cloquet's canal or vitreous cortex and gel over the posterior retina (Figure 9). To precisely diagnose the absence of a PVD, slit-lamp biomicroscopy should be performed in addition to the OCT examination. In many cases, it is impossible to diagnose the absence of a PVD using only OCT. At first, an age-related shallow PVD develops around the macula, which then progresses to one of three types: a shallow PVD without formation of a premacular hole in the posterior vitreous cortex, a shallow PVD with formation of a premacular hole in the posterior vitreous cortex with vitreous gel attachment to the macula, or a shallow PVD with formation of a premacular hole in the posterior vitreous cortex without vitreous gel attachment to the macula (Figure 10). The primary type of age-related shallow PVD, a perimacular shallow PVD, is seen frequently; the latter two types of shallow PVD are rarely observed because the liquefied vitreous enters the subhyaloid space through the premacular hole in the posterior vitreous cortex then induces the rapid formation of a high PVD, which can be detected by slit-lamp biomicroscopy but not OCT (Figure 11).

A perifoveal PVD with pinpoint vitreous traction to the fovea is seen frequently in cases of idiopathic macular holes. The foveal retina is very thin and can be punched out easily with weak vitreous traction. During macular-hole progression, liquefied vitreous is initially trapped in the subhyaloid space and pushes the posterior vitreous cortex anteriorly to induce the pinpoint vitreous traction to the fovea (Figure 12). A premacular hole in the posterior hyaloid membrane observed in an agerelated shallow PVD seems to be protective of the thin fragile
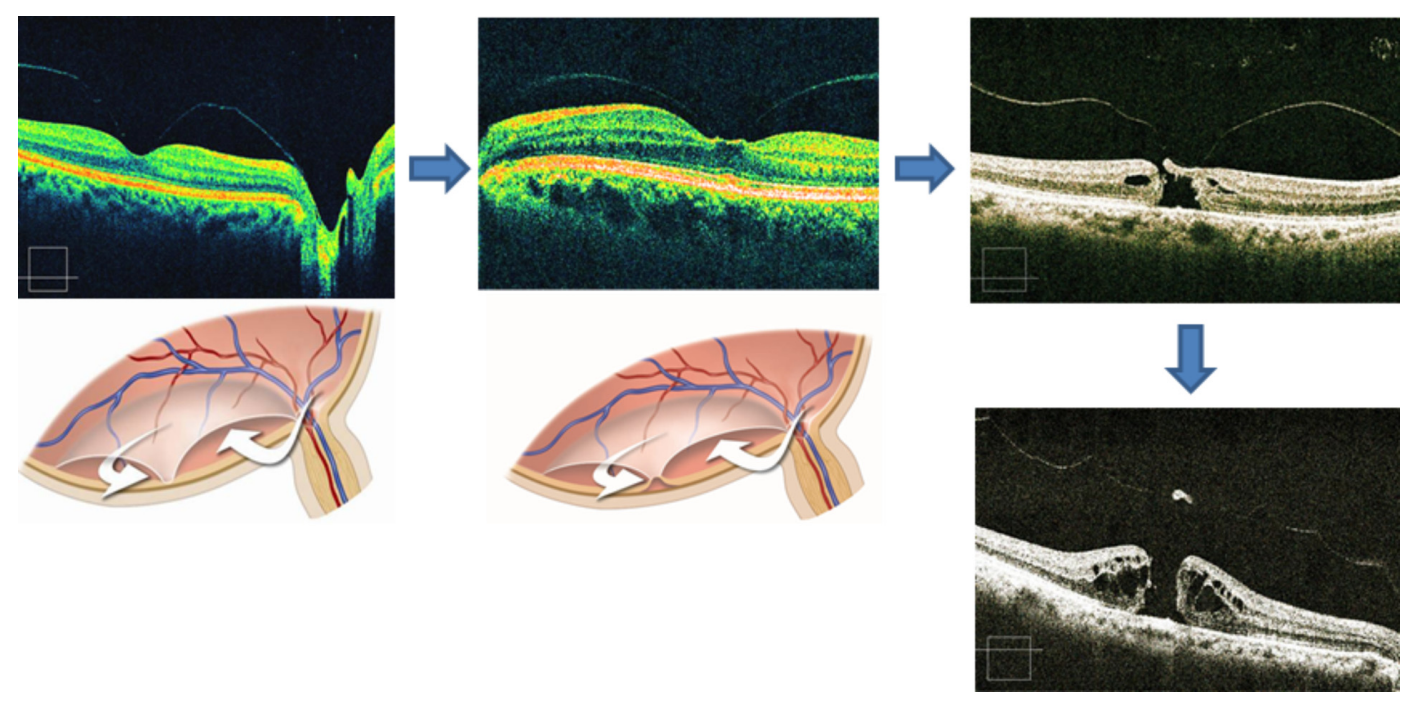

Figure 12 A shallow posterior vitreous detachment (PVD) without shrinkage of the posterior vitreous cortex. A perifoveal PVD associated with a macular hole. In the primary stage of a macular hole (top left optical coherence tomography [OCT] image), liquefied vitreous (arrows in the bottom left diagram) is trapped in the subhyaloid space and pushes the posterior vitreous cortex anteriorly to induce the pinpoint vitreous traction at the fovea (top middle OCT image and bottom right diagram) and then induces a macular tear (top right OCT image). Finally, a full-thickness macular hole develops with an operculum associated with a shallow PVD throughout the posterior pole in the late-stage macular hole (bottom right OCT image).

Note: Copyright @ 201 I, Dove Medical Press. Reproduced with permission from Takezawa M et al. Clarifying the mechanism of idiopathic macular hole development in fellow eyes using spectral-domain optical coherence tomography. Clin Ophthalmol. 201 I;5:10I-108. ${ }^{4}$ 

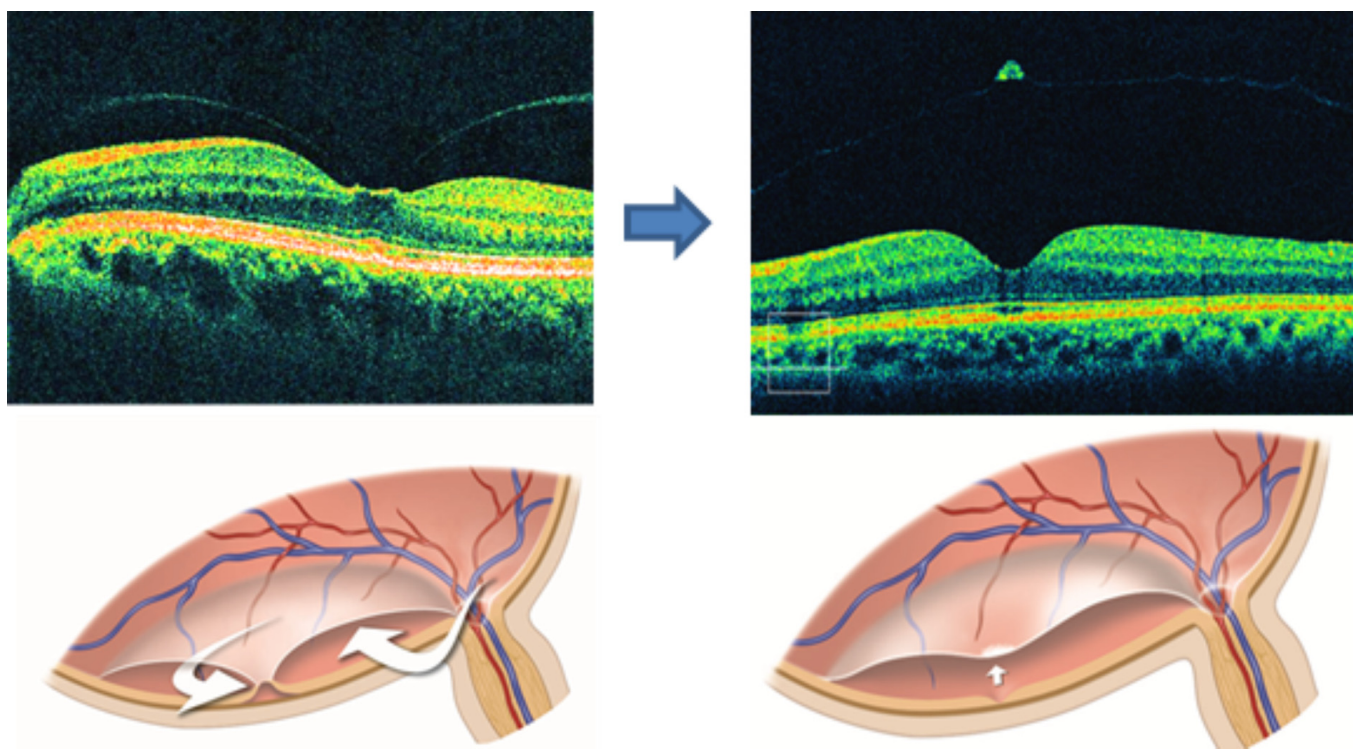

Figure I 3 Abortion of a macular hole. A stage I macular hole (top left optical coherence tomography image) forms as a result of vitreous traction on the fovea by trapped liquefied vitreous (arrows in the bottom left diagram) in the subhyaloid space. When a shallow posterior vitreous detachment develops with a pseudo-operculum (arrow in the bottom right diagram) throughout the posterior pole of the retina, the macular hole is aborted.

Note: Copyright $@ 201$ I, Dove Medical Press. Reproduced with permission from Takezawa M et al. Clarifying the mechanism of idiopathic macular hole development in fellow eyes using spectral-domain optical coherence tomography. Clin Ophthalmol. 20I I;5:10I-108. ${ }^{4}$
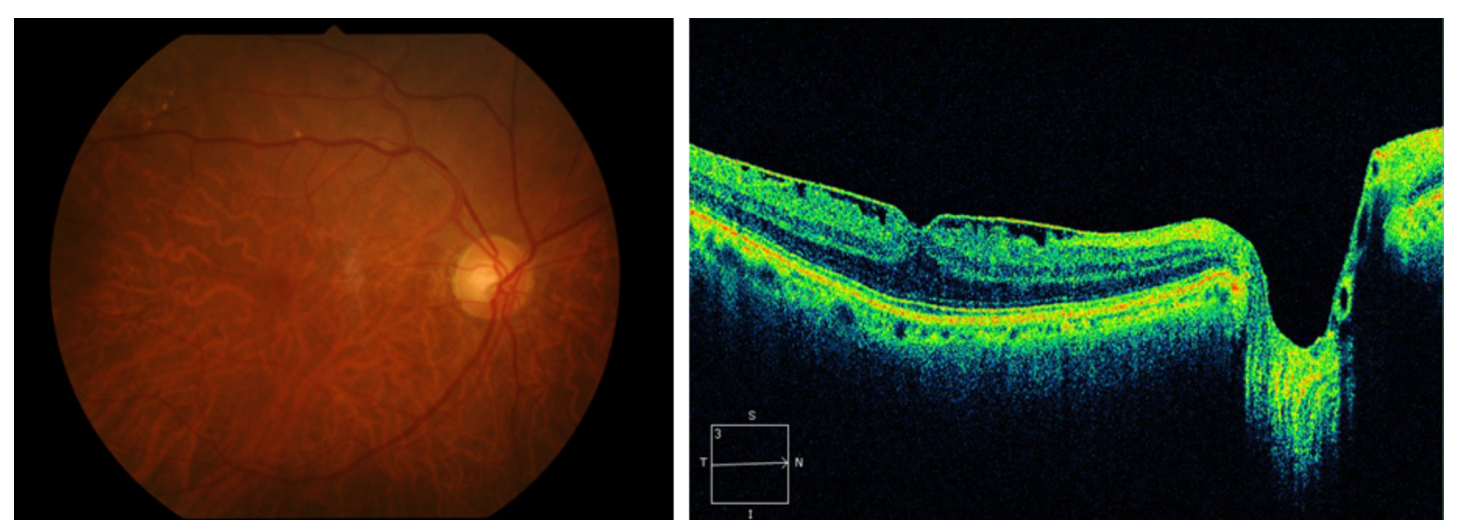

Figure I4 A shallow posterior vitreous detachment (PVD) with shrinkage of the posterior vitreous cortex in a case with an epiretinal membrane (ERM). Most cases of ERM have a shallow PVD with shrinkage of the posterior vitreous cortex.

A

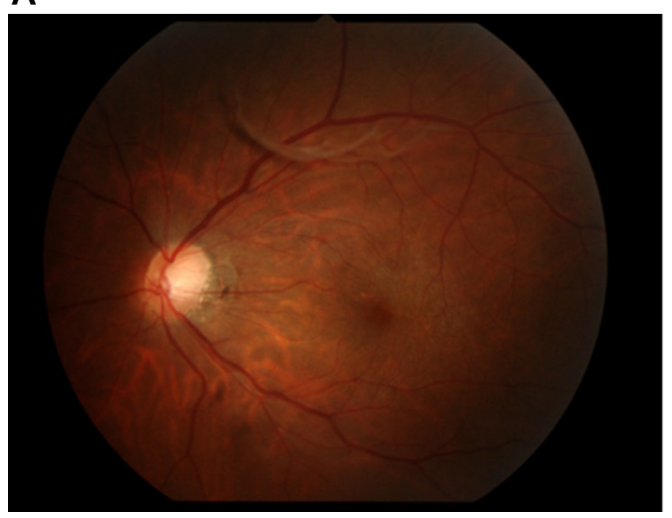

B

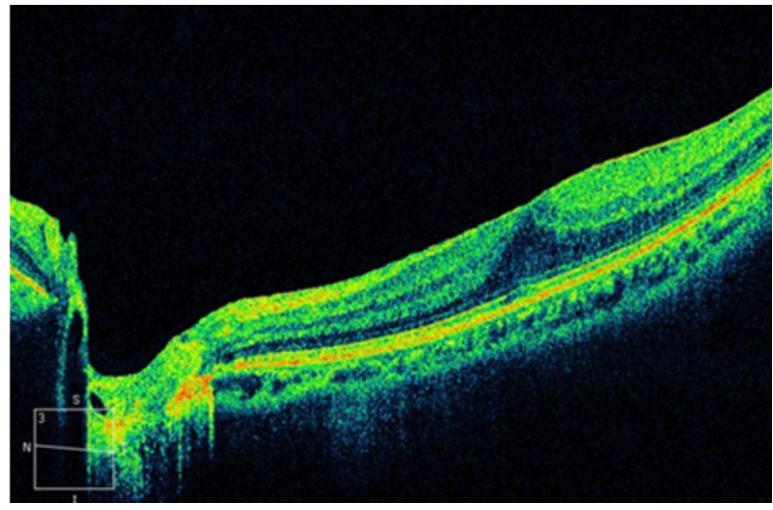

Figure 15 A partial posterior vitreous detachment (P-PVD) (M) and absence of a shallow posterior vitreous detachment (PVD). The color fundus photograph (A) shows an epiretinal membrane (ERM) and vitreous opacity associated with a P-PVD (M). However, no shallow PVD is found in this case of an ERM (B).

Abbreviation: $M$, vitreous attachment to the macula. 
A

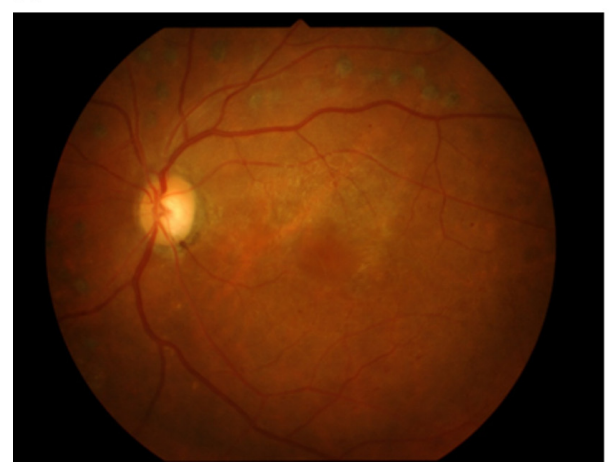

B

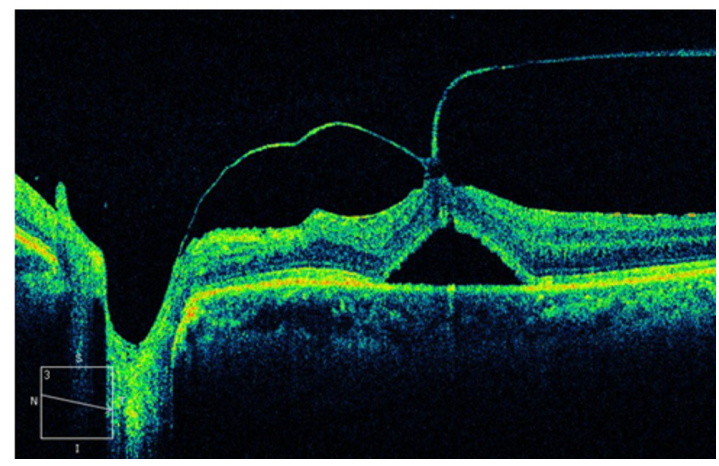

Figure 16 Vitreomacular traction syndrome. In a case with an advanced epiretinal membrane (A), a shallow posterior vitreous detachment with shrinkage of the posterior vitreous cortex causes a tractional retinal detachment at the fovea (B).
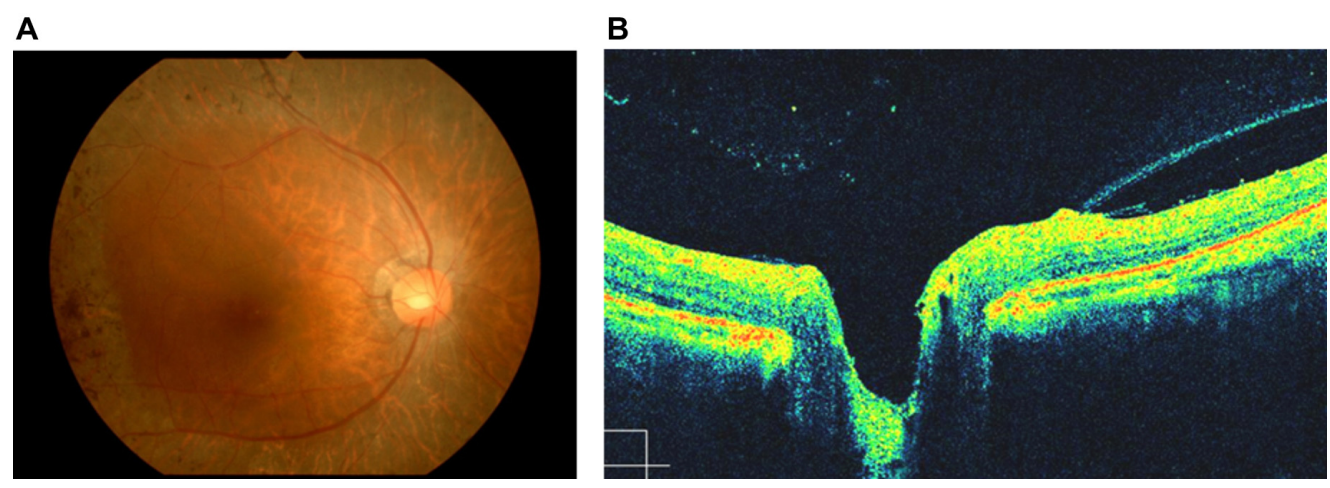

Figure 17 A case of retinitis pigmentosa shows typical bone spicule-shaped pigment deposits in the mid periphery (A) and posterior vitreoschisis in nasal fundus. A case of retinitis pigmentosa showing posterior vitreoschisis in the nasal fundus. A dense vitreous membrane due to posterior vitreoschisis in front of the retina and some residual cortex on the surface of retina are clearly seen in the optical coherence tomography image (B).

Note: Copyright (c) 20II, Dove Medical Press. Reproduced with permission from Takezawa M et al. Clarifying the mechanism of idiopathic macular hole development in fellow eyes using spectral-domain optical coherence tomography. Clin Ophthalmol. 20I I;5:10I-108. ${ }^{4}$

\section{A}

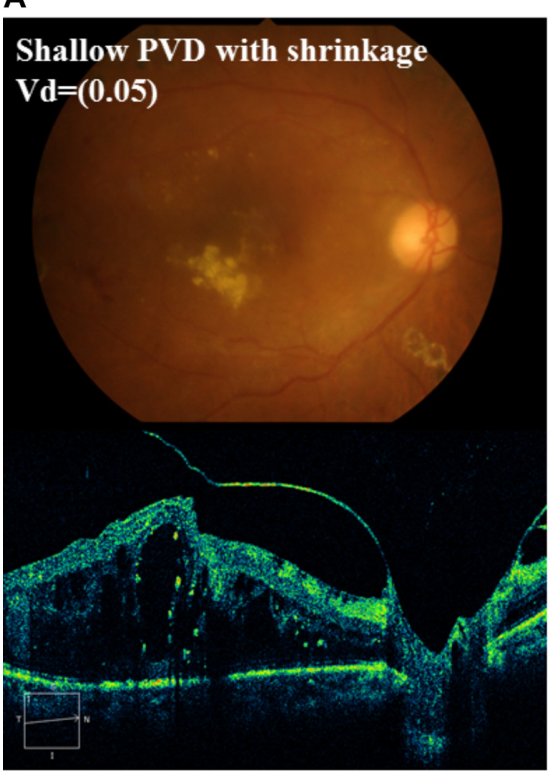

\section{B}

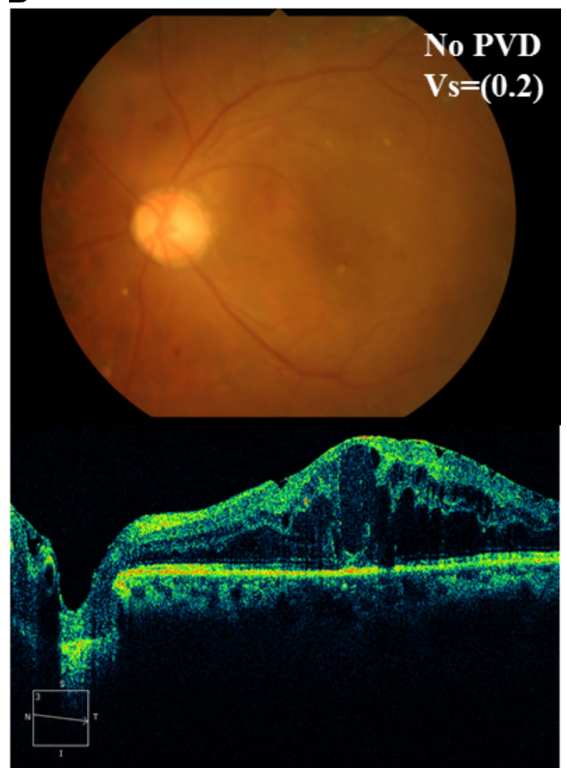

Figure 18 Diabetic macular edema (DME) with a shallow posterior vitreous detachment (PVD) that has shrinkage of the posterior vitreous cortex. DME is present bilaterally. The right eye (A) has more extensive deposition of hard exudates and decreased vision compared with the left eye (B). The right eye has a shallow PVD with shrinkage, but the left eye does not.

Notes: $\mathrm{Vd}=(0.05) ; \mathrm{Vs}=(0.2)$

Abbreviations: VD, visus dexter; VS ,visus sinister. 


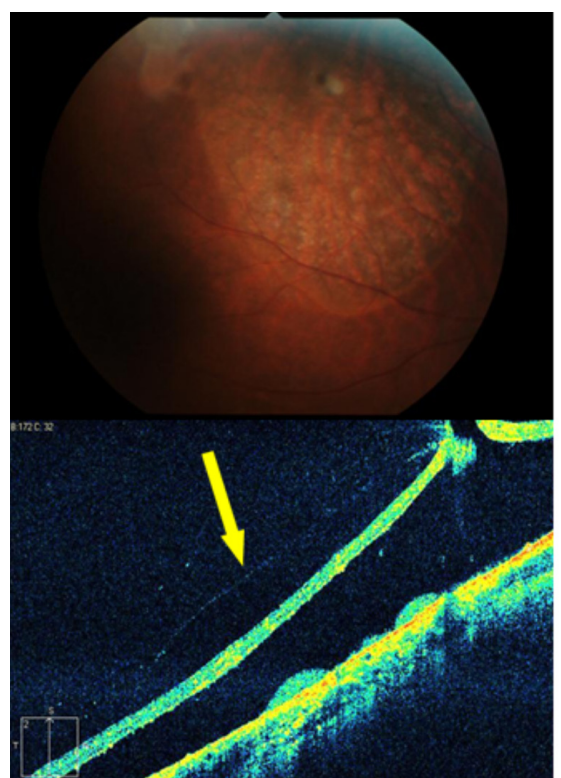

Before scleral buckling surgery

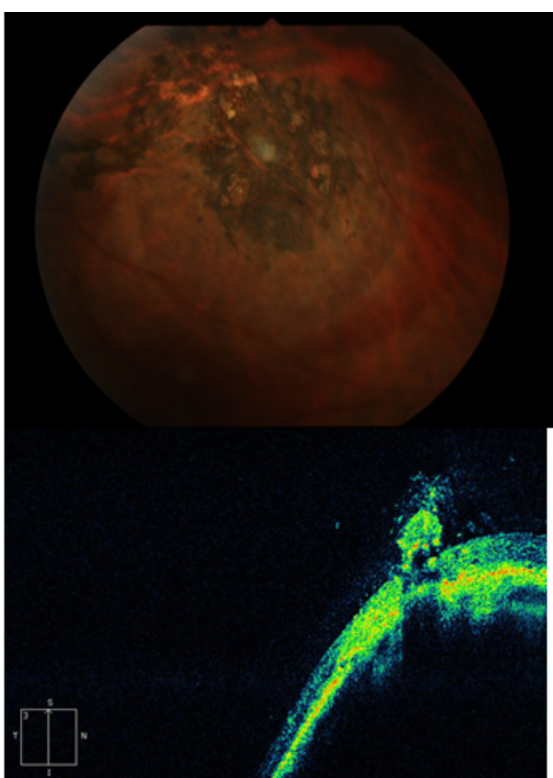

After scleral buckling surgery

Figure 19 A retinal break without evidence of a posterior vitreous detachment (PVD) biomicroscopically. A shallow retinal detachment with a horseshoe tear (top left fundus photo) is seen in the superior temporal quadrant in a 30-year-old man. Slit-lamp biomicroscopy does not show the absence of a PVD, but optical coherence tomography (OCT) shows a peripheral shallow PVD (arrow in the bottom left OCT image) exerted on the retinal break. After scleral buckling surgery, the detached retina is reattached by the effect of scleral indentation by the scleral buckling (right fundus photo and OCT image).

fovea from the vitreous traction. A full-thickness macular hole is observed with an operculum associated with a shallow PVD throughout the posterior pole in a late-stage macular hole (classic Gass stage 3). ${ }^{10}$ In some cases, abortion of the macular hole can occur in association with vitreofoveal separation in the early stage $^{11}$ (stage 1 or 2) (Figure 13). ${ }^{12}$ Therefore, a macular hole does not develop in the eye of patients with a macular hole in their other eye when there is a shallow PVD over the fovea, even if there is no evidence of PVD by biomicroscopy. ${ }^{4}$

A shallow PVD with shrinkage of the posterior vitreous cortex affects the macular function in many vitreoretinal diseases. Most cases of epiretinal membrane (ERM) have a shallow PVD with shrinkage of the posterior vitreous cortex (Figure 14). In some ERM cases, a shallow PVD is not present (Figure 15). In advanced cases of ERM, a tractional retinal detachment due to vitreomacular traction syndrome can develop (Figure 16). In such cases, vitrectomy should be performed. The posterior vitreoschisis is sometimes observed by OCT. We have previously reported that this pathology is observed by OCT in some cases of retinitis pigmentosa. ${ }^{13}$ Although posterior vitreoschisis can be confused with a shallow PVD with shrinkage of the posterior vitreous cortex, it is important that it be distinguished from this. The major difference can be seen by comparing the case with posterior vitreoschisis with the case with a shallow PVD with shrinkage of the posterior vitreous cortex shown in Figure 17; in the former, some cortex can be seen on the retinal surface while this is not visible in the latter. In certain cases with DME, vitreous traction on the macula due to a shallow PVD with shrinkage of the posterior vitreous cortex worsens the macular edema (Figure 18). In such cases, vitreous surgery is more effective than laser treatment or intravitreous injection of an anti-vascular endothelial growth factor drug or triamcinolone to treat the DME.

Some cases of retinal tears have no evidence of a PVD on biomicroscopy. In such cases, a peripheral shallow PVD is seen on OCT. A retinal tear can develop from a peripheral shallow PVD (Figure 19), and scleral buckling is more appropriate than vitrectomy in such cases.

\section{Conclusion}

The classification of PVD by slit-lamp biomicroscopy and classification of shallow PVD by OCT contribute to predicting the prognosis and determining the indication for vitreoretinal surgery in many vitreoretinal diseases.

\section{Disclosure}

The authors have no financial interest in any aspect of this report and declare no conflicts of interest in this work.

\section{References}

1. Kakehashi A, Kado M, Akiba J, Hirokawa H. Variations of posterior vitreous detachment. Br J Ophthalmol. 1997;81(7):527-532. 
2. Ono R, Kakehashi A, Yamagami H, et al. Prospective assessment of proliferative diabetic retinopathy with observations of posterior vitreous detachment. Int Ophthalmol. 2005;26(1-2):15-19.

3. Ophir A, Martinez MR. Epiretinal membranes and incomplete posterior vitreous detachment in diabetic macular edema, detected by spectraldomain optical coherence tomography. Invest Ophthalmol Vis Sci. 2011;52(9):6414-6420

4. Takezawa M, Toyoda F, Kambara C, Yamagami H, Kakehashi A. Clarifying the mechanism of idiopathic macular hole development in fellow eyes using spectral-domain optical coherence tomography. Clin Ophthalmol. 2011;5:101-108.

5. Kakehashi A. Examination technique of vitreous biomicroscopy. In: Schepens CL, Trempe CL, Takahashi M, editors. Atlas of Vitreous Biomicroscopy. Boston: Butterworth Heinemann; 1999:25-45.

6. Sebag J, Balazs EA. Morphology and ultrastructure of human vitreous fibers. Invest Ophthalmol Vis Sci. 1989;30(8):1867-1871.

7. Kicova N, Bertelmann T, Irle S, Sekundo W, Mennel S. Evaluation of a posterior vitreous detachment: a comparison of biomicroscopy, B-scan ultrasonography and optical coherence tomography to surgical findings with chromodissection. Acta Ophthalmol. 2012;90(4):e264-e268.
8. Uchino E, Uemura A, Ohba N. Initial stages of posterior vitreous detachment in healthy eyes of older persons evaluated by optical coherence tomography. Arch Ophthalmol. 2001;119(10):1475-1479.

9. Johnson MW. Posterior vitreous detachment: evolution and complications of its early stages. Am J Ophthalmol. 2010;149(3):371-382. e1.

10. Gass JD. Idiopathic senile macular hole: its early stages and pathogenesis. Arch Ophthalmol. 1988;106(5):629-639.

11. Gass JD, Van Newkirk M. Xanthic scotoma and yellow foveolar shadow caused by a pseudo-operculum after vitreofoveal separation. Retina. 1992;12(3):242-244.

12. Kakehashi A, Schepens CL, Akiba J, Hikichi T, Trempe CL. Spontaneous resolution of foveal detachments and macular breaks. Am JOphthalmology. 1995;120(6):767-775.

13. Takezawa M, Tetsuka S, Kakehashi A. Tangential vitreous traction: a possible mechanism of development of cystoid macular edema in retinitis pigmentosa. Clin Ophthalmol. 2011;5:245-248.

\section{Clinical Ophthalmology}

\section{Publish your work in this journal}

Clinical Ophthalmology is an international, peer-reviewed journal covering all subspecialties within ophthalmology. Key topics include: Optometry; Visual science; Pharmacology and drug therapy in eye diseases; Basic Sciences; Primary and Secondary eye care; Patient Safety and Quality of Care Improvements. This journal is indexed on Submit your manuscript here: http://www.dovepress.com/clinical-ophthalmology-journal

\section{Dovepress}

PubMed Central and CAS, and is the official journal of The Society of Clinical Ophthalmology (SCO). The manuscript management system is completely online and includes a very quick and fair peer-review system, which is all easy to use. Visit http://www.dovepress.com/ testimonials.php to read real quotes from published authors. 